\title{
Synthesis, photophysical and metal ion signalling behaviour of mono- and di-azacrown derivatives of 4 -aminophthalimide
}

\author{
N B SANKARAN ${ }^{\mathrm{a}}$, M SARKAR and A SAMANTA* \\ School of Chemistry, University of Hyderabad, Hyderabad 500 046, India \\ ${ }^{a}$ Present address: Department of Chemistry, Graduate School of Science, Tohoku University, Sendai, Japan \\ e-mail: assc@uohyd.ernet.in
}

\begin{abstract}
Synthesis, photophysical behaviour and metal ion signalling ability of 3-component systems, I and II, comprising a 4-aminophthalimido moiety as fluorophore, a dimethylene spacer and two different azacrown receptors, are reported. The fluorescence quantum yields and lifetimes of both the systems have been found to be significantly lower than that of the parent fluorophore indicating the occurrence of photoinduced electron transfer (PET) between the terminal moieties. PET is found to be more efficient in II, presumably due to the presence of more than one electron-donating centre in the receptor moiety. Fluorescence decay behaviour of the systems suggests a through-space nature of PET. The systems exhibit off-on fluorescence signalling in the aprotic media in the presence of several metal ions, some of which are well known for their fluorescence quenching abilities. Diazacrown derivative, II, appears to be a somewhat better signalling system than the monoazacrown derivative, $\mathbf{I}$.
\end{abstract}

Keywords. 4-Aminophthalimide; photophysical behaviour; fluorescence signalling; metal ions; crown compounds.

\section{Introduction}

In recent years, there has been a great deal of interest in molecular systems capable of performing light-induced logic operations. ${ }^{1-3}$ A variety of molecular photonic devices, which have been developed in recent years, are essentially assemblies of suitably organized molecular components that perform logic functions characteristic of the assembly. ${ }^{4-12}$ Of particular interest are the fluorosensors, molecular systems that are powered by light and whose response to the guest molecule or ion is based on fluorescence, for various metal ions of environmental and biological interest.

Fluorosensors are most often three-component systems consisting of a signalling moiety, called fluorophore and a guest-binding site, commonly referred to as receptor; the two are usually separated by a spacer group. ${ }^{4}$ The components are chosen such that communication between the receptor and fluorophore results in 'switching off' of the fluorescence of the system. However, the fluorescence is 'switched on' in the presence of a guest, which turns off the communication between the receptor and fluorophore. Thus, the presence of a guest is indi-

Dedicated to the memory of the late Professor Bhaskar G Maiya *For correspondence cated by fluorescence enhancement (FE) of the system. Photoinduced electron transfer (PET) is the most commonly exploited mechanism of communication between the terminal moieties of a fluorophorespacer-receptor system. ${ }^{4}$ The design principle of the 'off-on' fluorescence signalling of a guest is schematically illustrated in scheme 1 .

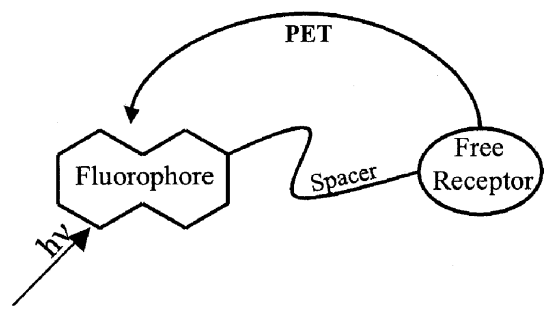

'Off' state

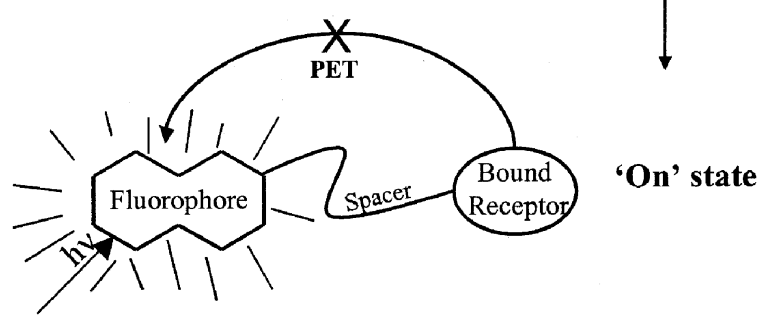

Scheme 1. 
Sensing of the metal ions, particularly the transition and heavy metal ions, is of considerable interest for various reasons. ${ }^{13-15}$ The crown compounds, because of the macrocyclic effect, expect to show stronger binding of the guests and to display selectivity in binding, depending on their cavity size., ${ }^{3,16,17}$ Though the oxygen crowns bind the $s$-block metal ions, these are not good coordinating ligands for the transition metal ions. When the oxygen atom of the crown ether is replaced by nitrogen, one can expect stronger binding of the transition metal ions because of the enhanced binding ability of these metal ions with nitrogen. ${ }^{18}$ Keeping this in mind and in continuation of our studies on systems capable of signalling metal ions through fluorescence enhancement, ${ }^{19-26}$ we have developed fluorophore-spacer-receptor systems, I and II (chart 1). The fluorophore component used in these systems is 4-aminophthalimide (AP), whose photophysical behaviour is well understood. ${ }^{27-29}$ Moreover, since AP is an electron-deficient molecule, it is ideally suited as the fluorophore component of the fluorophore-spacer-receptor sensor system. ${ }^{19,22}$ In the present systems, two different azacrown moieties have been used as the receptor to examine the effect of the number of electron-donating centres on the signalling efficiencies. The synthetic procedure, photophysical properties and metal ion signalling behaviour of the two systems in tetrahydrofuran (THF) and acetonitrile (AN) are presented in this paper.

\section{Experimental}

\subsection{Materials}

Reagent grade reactants and solvents were obtained from commercial suppliers and used as received for synthesis. The solvents used for absorption and fluorescence studies were purified by standard proce-

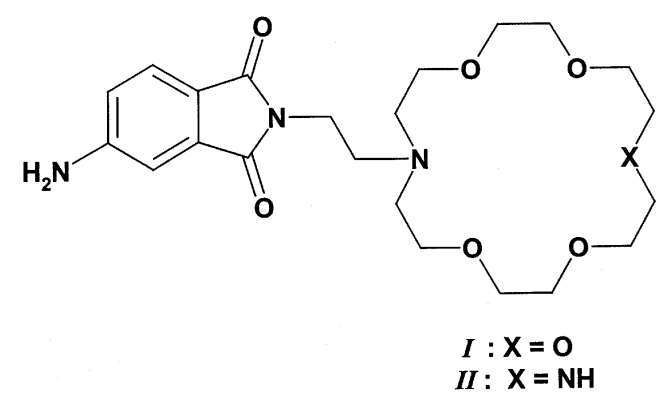

Chart 1. dures. ${ }^{30}$ Hydrated perchlorate salts of the metals (Aldrich or Acros Organics Ltd.) were used throughout the investigation except in the case of mercury, whose chloride salt, mercuric chloride, was used. Starting materials required for the synthesis of $\mathbf{I}$ and II were prepared as described in the literature. ${ }^{26,31,32}$

2.1a Synthesis of 5-amino-2-[2-(1,4,7,10,13-pentaoxa-16-aza-cyclooctadec-16-yl)-ethyl]-isoindole-1,3dione (I): 5-Amino-2-(2-bromoethyl)-isoindole-1,3dione $(0.5 \mathrm{~g}, 1.9 \mathrm{mmol})$ and the crown ether, 1,4,7,10,13-pentaoxa-16-aza-cyclooctadecane (2 g, $7.6 \mathrm{mmol}$ ) were stirred in acetonitrile at reflux temperature in the presence of potassium carbonate $(1 \mathrm{~g}, 7.2 \mathrm{mmol})$ for $24 \mathrm{~h}$. The reaction mixture was filtered, concentrated, washed with water and extracted using ethylacetate. The organic layer was dried using anhydrous sodium sulphate and reduced in volume. The crude solid obtained was purified using a neutral alumina column with $2 \%$ methanol in chloroform as the eluent. Yield: $0.4 \mathrm{~g} \mathrm{(47.63 \% ).}$ IR (neat, $\mathrm{cm}^{-1}$ ): 3417, 2980, 2873, 1701, 1616, 1461, 1355, 1110, 952, 839, ${ }^{1} \mathrm{H}$ NMR $\left(\mathrm{CDCl}_{3}, 200 \mathrm{MHz}\right)$ : $\delta 2.7(m, 8 \mathrm{H}), 3.6(m, 20 \mathrm{H}), 6.8(d, 1 \mathrm{H}), 7.05(s, 1 \mathrm{H})$, $7.4(d, 1 \mathrm{H})$.

2.1b Synthesis of 5-amino-2-[2-(1,4,10,13-tetraoxa-7,16-diaza-cyclooctadec-7-yl)-ethyl]-isoindole-1, 3-dione (II): 5-Amino-2-(2-bromoethyl)-isoindole1,3-dione $(0.45 \mathrm{~g}, 1.7 \mathrm{mmol})$ and the diazacrown ether, 1,4,10,13-tetraoxa-7,16-diaza-cyclooctadecane $(2 \mathrm{~g}, 7.6 \mathrm{mmol})$ were mixed in acetonitrile $(25 \mathrm{~mL})$ and stirred well in the presence of potassium carbonate $(1 \mathrm{~g}, 7.2 \mathrm{mmol})$ under refluxing conditions. The reaction was continued for $24 \mathrm{~h}$. The reaction mixture was filtered, concentrated, washed with water and extracted using ethylacetate. The organic layer was dried using anhydrous sodium sulphate and reduced in volume. The resulting solid was purified by column chromatography using a neutral alumina column and the required fraction was obtained with a $2 \%$ methanol in chloroform as eluent. Yield: $0.5 \mathrm{~g}$ $(66.4 \%)$. Melting point: $142^{\circ} \mathrm{C}$, IR $\left(\mathrm{KBr}, \mathrm{cm}^{-1}\right)$ : 3485, 2893, 2793, 1753, 1701, 1597, 1458, 1390, 1111, 987, ${ }^{1} \mathrm{H}$ NMR $\left(\mathrm{CDCl}_{3}, 200 \mathrm{MHz}\right): \delta 2.7(m$, $12 \mathrm{H}), 3 \cdot 5(\mathrm{~m}, 16 \mathrm{H}), 6 \cdot 8(d, 1 \mathrm{H}), 7 \cdot 05(s, 1 \mathrm{H}), 7 \cdot 4(d$, $1 \mathrm{H})$, FAB-MS $m / z: 451[M+1]^{+}$.

Three-component systems, I and II, as stated above, were purified by column chromatography. Production of a single spot in TLC, which is an indicator of the purity of a sample, was checked prior to fluorescence measurements. 


\subsection{Instrumentation and methods}

Steady state absorption and fluorescence spectra were recorded on a UV-Vis-NIR spectrophotometer (Shimadzu, UV-3101PC) and spectrofluorimeter (Spex, Fluoromax 3) respectively. NMR spectra were recorded on a Bruker ACF-200 spectrometer, the FAB mass spectra on a JEOL SX 102/DA-6000 mass spectrometer, and IR spectra on a Jasco FTIR/5300 spectrometer. Fluorescence decay measurements were performed using a single-photon counting spectrofluorimeter (IBH, model 5000). The instrument was equipped with a thyratron-gated coaxial flash lamp filled with hydrogen gas at $0.5 \mathrm{~atm}$ pressure. The lamp frequency was maintained at $40 \mathrm{kHz}$ and the FWHM of the lamp pulse under the operating condition was $\sim 1.2 \mathrm{~ns}$. The decay curves were analysed by a nonlinear least squares iteration procedure using IBH decay analysis software (version $6 \cdot 1 \cdot 36$ ). The quality of the fit was judged by the $\chi^{2}$ values and plot of the residuals.

Fluorescence quantum yields of the systems were measured using 4-aminophthalimide as the reference compound $\left(\phi_{f}=0.63\right.$ in acetonitrile) ${ }^{29}$ A solution of the systems in tetrahydrofuran/acetonitrile was prepared with absorbance (between 0.05 and 0.1 ) being the same as that of the reference compound at the exciting wavelength $(\lambda=360 \mathrm{~nm})$. Fluorescence spectra of the systems and the reference compound were measured under the same operating conditions and settings and the quantum yields were determined by comparing the areas beneath the fluorescence spectra.

Fluorescence measurements were carried out using $\sim 10^{-5} \mathrm{M}$ solutions of the compounds. The effect of the metal ions on the fluorescence intensity was examined by adding a few microlitres of the stock solution of the metal salts to a known volume of the solution of the sensor system $(3 \mathrm{ml})$. The addition was limited to $100 \mu \mathrm{l}$ such that the volume change was not significant.

\section{Results and discussion}

\subsection{Photophysical behaviour}

Both systems exhibit broad absorption bands (figure 1) typical of the intramolecular charge transfer (ICT) transition of the 4-aminophthalimide moiety ${ }^{22,27,29}$ except that the spectral maxima for the crown derivatives are slightly red-shifted relative to that of the parent system, AP (vide table 1) in any given solvent. The red-shift of the absorption maximum of the multi-component systems relative to that of the parent molecule is most likely due to the greater polar characteristic of the substituted fluorophore rather than due to any interaction between the terminal moieties of the molecules. As can be seen from the spectral data shown in table 1 , the variation of $\lambda_{\max }^{a b s}$ of the two systems in any given medium is too small to comment upon. The $\lambda_{\max }^{a b s}$ values are marginally red-shifted in polar solvents. This behaviour is again consistent with that observed with the parent fluorophore, 4-aminophthalimide.

The crown compounds exhibit a broad structureless emission band (figure 1) characteristic of the ICT emission of AP with the $\lambda_{\max }^{f l u}$ Stokes-shifted relative to the parent molecule (table 1). Fluorescence spectral data show that the variation of $\lambda_{\max }^{f l u}$ among the different derivatives is quite small. However, unlike the absorption, the fluorescence maxima of the systems exhibit significant shift to longer wavelengths with increase in the polarity of
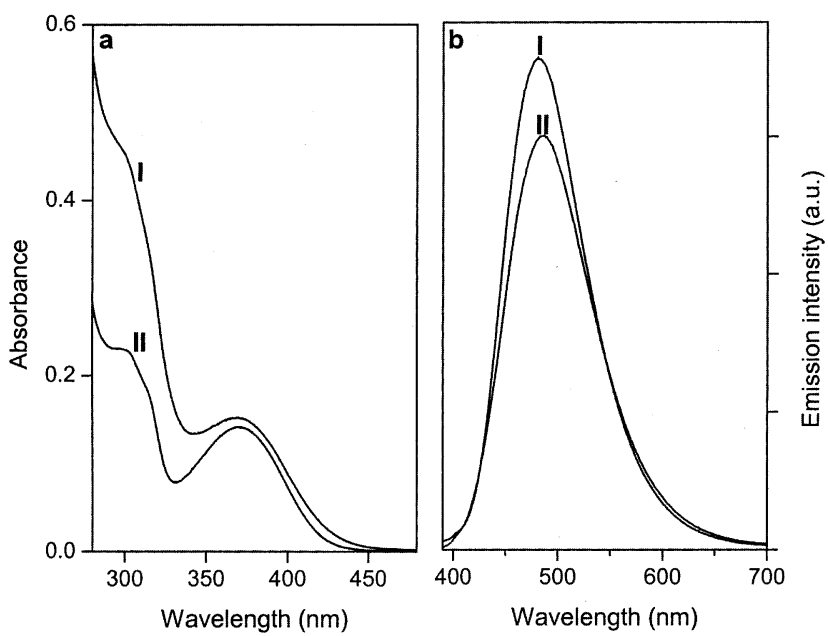

Figure 1. Absorption (a) and fluorescence (b) spectra of $\mathbf{I}$ and $\mathbf{I I}$ in AN, $\lambda_{\mathrm{exc}}=360 \mathrm{~nm}$.

Table 1. Wavelength (nm) $)^{\mathrm{a}}$ corresponding to the absorption and emission maxima ${ }^{b}$ of the systems.

\begin{tabular}{llllll}
\hline & \multicolumn{2}{c}{ THF } & & \multicolumn{2}{c}{ AN } \\
\cline { 2 - 3 } \cline { 5 - 6 } Compound & $\lambda_{\text {abs }}$ & $\lambda_{\text {em }}$ & & $\lambda_{\text {abs }}$ & $\lambda_{\text {em }}$ \\
\hline AP & 358 & 452 & & 359 & 462 \\
I & 369 & 468 & & 370 & 480 \\
II & 367 & 483 & & 370 & 484 \\
\hline
\end{tabular}

${ }^{\mathrm{a}} \pm 1 \mathrm{~nm} ;{ }^{\mathrm{b}} \lambda_{\mathrm{exc}}=360 \mathrm{~nm}$ 
Table 2. Fluorescence quantum yield $\left(\phi_{f}\right)^{\mathrm{a}}$ and decay parameters $(\tau \text {, in } \mathrm{ns})^{\mathrm{b}}$ for the systems. The relative weightage of each decay component (in \%) is given in brackets.

\begin{tabular}{|c|c|c|c|c|c|c|}
\hline \multirow[b]{2}{*}{ System } & \multicolumn{3}{|c|}{ In THF } & \multicolumn{3}{|c|}{ In AN } \\
\hline & $\phi_{f}$ & $\tau_{f}$ & $\tau_{a v}$ & $\phi_{f}$ & $\tau_{f}$ & $\tau_{a v}$ \\
\hline $\mathrm{AP}^{\mathrm{c}}$ & 0.70 & $14 \cdot 0$ & $14 \cdot 0$ & 0.63 & 12.4 & $12 \cdot 4$ \\
\hline I & $0 \cdot 25$ & $1.9(35), 9 \cdot 3(16 \cdot 4), 17 \cdot 3(48 \cdot 6)$ & $10 \cdot 6$ & $0 \cdot 24$ & $0 \cdot 2(78), 3 \cdot 8(8 \cdot 9), 18 \cdot 7(13 \cdot 1)$ & $2 \cdot 9$ \\
\hline II & 0.04 & $0 \cdot 9(71), 4 \cdot 1(24), 17 \cdot 8(5)$ & $2 \cdot 5$ & 0.03 & $0.3(89.7), 1.7(8.7), 16.3(1.6)$ & 0.6 \\
\hline
\end{tabular}

${ }^{\mathrm{a}} \pm 10 \% ;{ }^{\mathrm{b}} \pm 10 \%$ for lifetimes more than $1 \mathrm{~ns}, \tau$ values lower than $1 \mathrm{~ns}$ may not be accurate because of the time resolution of the set up; ${ }^{c}$ these values are taken from ref. [29]

the medium. This behaviour is consistent with the greater polar nature of the fluorescent state of AP and its derivatives. ${ }^{29}$

While the spectral behaviour of the crown compounds is very similar to that of AP, the fluorescence quantum yields $\left(\phi_{f}\right)$ of these systems are found significantly lower than that of AP (table 2). AP is highly fluorescent in aprotic media with fluorescence quantum yield between 0.63 and $0.73 .{ }^{29}$ The $\phi_{f}$ values for I and II are $\sim 0.24$ and 0.04 respectively. The lower fluorescence quantum yield of crown derivatives is indicative of PET interaction between the terminal fluorophore and receptor moieties in these systems. The solvent effect on PET, as observable from the $\phi_{f}$ values, is negligible.

The fluorescence decay parameters of the systems in THF and AN have been collected in table 2. Even though AP exhibits a single exponential decay with a long lifetime of 12.4-14 ns in aprotic media, both I and II exhibit tri-exponential decay behaviour with the average lifetime significantly shorter than that of AP. Among the three components, the major component is associated with short (sub-nanosecond) lifetime. The second component is characterized by long lifetime similar to that of the parent fluorophore. The third component has lifetime between that of the two other components. The lifetime values and the associated pre-exponential factors suggest that there exist at least three distinguishable conformations of each system. The short-component, which is the most dominant one, arises due to those molecules in which the orientation and distance between the interacting groups are most favourable for PET interaction. On the other hand, the component with the longest lifetime originates from those molecules in which the spatial disposition of the interacting groups is unfavourable for PET. This assignment and our earlier results ${ }^{19-24}$ on systems with much simpler receptor moieties suggest that the PET interaction is mediated by through-space inter- action between the fluorophore and the receptor moieties. The average fluorescence lifetime, which is one of the indicators of the extent of PET and is important from the point of view of fluorescence signalling, is relatively lower for the di-azacrown derivative, II. Interestingly, the lifetime values of the systems are relatively lower in polar solvent, AN.

\subsection{Effect of the metal ions}

Neither the extinction coefficient nor the wavelength of absorption changes significantly in the presence of the metal salts. A small red-shift of the absorption maximum $(\sim 5-7 \mathrm{~nm})$ could be observed with a large majority of the metal salts. In most of the cases, no isosbestic point could be observed.

The effect of the metal ions is however much more pronounced on the fluorescence behaviour of the systems. Addition of the metal salts leads to larger Stokes shift of the fluorescence maximum $(\sim 30$ $40 \mathrm{~nm})$ and is associated with an enhancement of the fluorescence intensity (figure 2). The maximum FE values observed for the systems are collected in table 3. As can be seen, the monoazacrown compound, I, exhibits very little FE in the presence of the metal ions. On the other hand, the diazacrown derivative, II, gives reasonably good enhancement in most of the cases. This behaviour is in agreement with our expectation based on the fluorescence lifetime of the individual compounds (table 2).

We have also examined the effect of the metal ions on the fluorescence decay behaviour of the systems. That the metal ions disrupt the PET communication between the terminal moieties of the systems is evident from the disappearance of the short component of the decay in the presence of the metal ions.

In summary, we have synthesised and fully characterised two new fluorophore-spacer-receptor systems consisting of two different azacrown moieties 

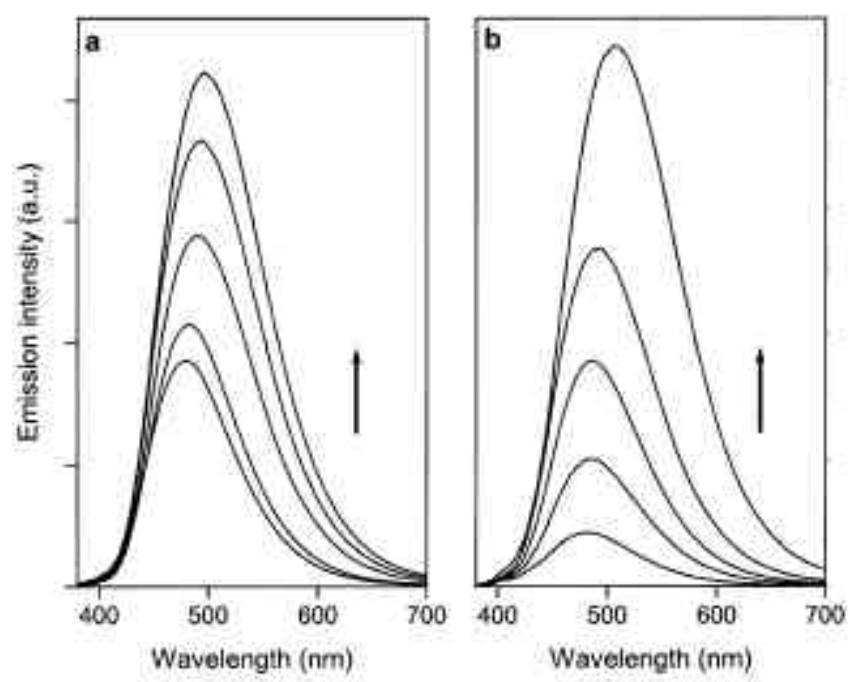

Figure 2. Effect of the addition of $\mathrm{Cd}^{2+}$ on the fluorescence behaviour of $\mathbf{I}(\mathbf{a})$ and $\mathbf{I I}(\mathbf{b})$ in $\mathrm{AN}, \lambda_{\mathrm{exc}}=360 \mathrm{~nm}$; $\left[\mathrm{Cd}^{2+}\right]$ (in $\mathrm{mM}$ ) were: $0,0 \cdot 12,0.28,0.87$ and 2.3 (in a) and $0,0 \cdot 036,0 \cdot 068,0.28$ and 0.7 (in b) respectively in the direction of the arrow.

Table 3. Maximum FE values ${ }^{\mathrm{a}}$ observed for the systems in the presence of the metal ions.

\begin{tabular}{lcccrr}
\hline & \multicolumn{2}{c}{$\mathbf{I}$} & & \multicolumn{2}{c}{ II } \\
\cline { 2 - 3 } \cline { 5 - 6 } Metal ion & THF & AN & & THF & AN \\
\hline $\mathrm{Cd}^{2+}$ & 2 & 3 & & 17 & 12 \\
$\mathrm{Co}^{2+}$ & 2 & 2 & & 5 & 17 \\
$\mathrm{Cu}^{2+}$ & $1 \cdot 6$ & $1 \cdot 3$ & & 3 & 9 \\
$\mathrm{Fe}^{3+}$ & $1 \cdot 6$ & $1 \cdot 4$ & & 5 & 8 \\
$\mathrm{Hg}^{2+}$ & $1 \cdot 5$ & $1 \cdot 2$ & & 2 & 3 \\
$\mathrm{Mn}^{2+}$ & 2 & 2 & & 10 & 4 \\
$\mathrm{Ni}^{2+}$ & 2 & 2 & & 4 & 13 \\
$\mathrm{~Pb}^{2+}$ & 2 & 2 & & 5 & 5 \\
$\mathrm{Zn}^{2+}$ & 2 & 3 & & 5 & 19 \\
\hline
\end{tabular}

${ }^{\mathrm{a}} \pm 15 \%$

as receptors and examined their photophysical and fluorescence signalling properties towards some metal ions of interest. The diazacrown derivative is found to show fairly good fluorescence enhancement even in the presence of quenching metal ions.

\section{Acknowledgements}

This work has been supported by the Department of Science and Technology (DST), Government of India and Council of Scientific and Industrial Research (CSIR). The authors also thank the UPE Program of the University Grants Commission (UGC) for some instrumental facilities.

\section{References}

1. Carter F L, Siatkowski R E and Wohltjen H (eds) 1988 Molecular electronic devices (Amsterdam: Elsevier)

2. Lehn J-M 1995 Supramolecular chemistry (Weinheim: $\mathrm{VCH}$ )

3. Steed J W and Atwood J L 2000 Supramolecular chemistry (New York: John Wiley \& Sons)

4. de Silva A P, Gunaratne H Q N, Gunnlaugsson T, Huxley A J M, McCoy C P J, Rademacher T and Rice T E 1997 Chem. Rev. 971515

5. Rurack K and Genger U R 2002 Chem. Soc. Rev. 31 116

6. Bissel R A, de Silva A P, Gunaratne H Q N, Lynch P L M, Maguire G E M and Sandanayake K R A S 1992 Chem. Soc. Rev. 21187

7. Bissel R A, de Silva A P, Gunaratne H Q N, Lynch P L M, Maguire G E M, McCoy C P and Sandanayake K R A S 1993 Top. Curr. Chem. 168223

8. Czarnik A W (ed.) 1993 In Fluorescent chemosensors for ion and molecule recognition (Washington DC: ACS), ACS Symposium Series 538

9. Balzani V (ed.) 1987 Supramolecular photochemistry (Dordrecht: Reidel)

10. Fabbrizzi L and Poggi A 1995 Chem. Soc. Rev. 24 197

11. Ghosh P, Bharadwaj P K, Mandal S and Ghosh S 1996 J. Am. Chem. Soc. 1181553

12. Ghosh P, Bharadwaj P K, Roy J and Ghosh S 1997 J. Am. Chem. Soc. 11911903

13. Fabbrizzi L, Lichelli M, Pallavicini P, Prodi L and Taglietti A 1999 Transition metals in supramolecular chemistry (ed.) J P Sauvage (New York: John Wiley \& Sons)

14. Yoon J, Ohler N, Vance E D H, Aumiller W D and Czarnik A W 1997 Tetrahedron Lett. 383845

15. Kimura E and Koike T 1998 Chem. Soc. Rev. 27179

16. Schneider H J and Yatsimirsky A K 2000 Principles and methods in supramolecular chemistry (New York: John Wiley \& Sons)

17. Pedersen C J 1967 J. Am. Chem. Soc. 897017

18. Hiraoka M 1982 Crown compounds: Their characteristics and applications (Amsterdam: Elsevier)

19. Ramachandram B and Samanta A 1997 Chem. Commun. 1037

20. Ramachandram B and Samanta A 1998 J. Phys. Chem. A102 10579

21. Ramachandram B and Samanta A 1998 Chem. Phys. Lett. 2909

22. Ramachandram B, Sankaran N B and Samanta A 1999 Res. Chem. Interm. 25843

23. Ramachandram B, Saroja G, Sankaran N B and Samanta A 2000 J. Phys. Chem. B104 11824

24. Ramachandram B, Sankaran N B, Karmakar R, Saha S and Samanta A 2000 Tetrahedron 567041 
25. Sankaran N B, Banthia S, Das A and Samanta A 2002 New J. Chem. 261529.

26. Banthia S and Samanta A 2002 J. Phys. Chem. B106 5572

27. Soujanya T, Krishna T S R and Samanta A $1992 \mathrm{~J}$. Photochem. Photobiol. A66 185

28. Soujanya T, Krishna T S R and Samanta A $1992 J$. Phys. Chem. 968544
29. Soujanya T, Fessenden R W and Samanta A 1996 J. Phys. Chem. 1003507

30. Perrin D D, Armarego W L F and Perrin D R 1980 Purification of laboratory chemicals (New York: Pergamon)

31. Maeda H, Nakatsuji Y and Okahara M 1981 Chem. Commun. 471

32. Bogatsky A V, Lukyaninko N G, Basok S S and Ostrovskaya L K 1984 Synthesis 138 